\title{
Time-Dependent Change in Omentin-1 Level Correlated with Early Improvement of Myocardial Function in Patients with First Anterior ST-Segment Elevation Myocardial Infarction After Primary Percutaneous Coronary Intervention
} \author{
and Yingxin Zhao ${ }^{1,2}$ \\ ${ }^{1}$ Department of Cardiology, Beijing Anzhen Hospital, Capital Medical University, Beijing, China \\ ${ }^{2}$ Beijing Institute of Heart Lung and Blood Vessel Disease, Beijing, China \\ ${ }^{3}$ Beijing Da Xing Hospital, Capital Medical University, Beijing, China
}

Yong Zhu ${ }^{1,2}$, Chengping $\mathrm{Hu}^{1,2}$, Yu Du ${ }^{1,2}$, Jianwei Zhang ${ }^{1,2}$, Jinxing Liu ${ }^{1,2}$, Guojie Cheng ${ }^{3}$, Hongya Han ${ }^{1,2}$

Aim: Omentin-1, as a novel adipocytokine, ameliorates obesity-associated disorders and suppresses the development of atherosclerotic lesions. The present research investigated the correlation between serum omentin-1 and post-infarction myocardial function.

Methods: A total of 52 patients with first anterior ST-segment elevation myocardial infarction (STEMI) were recruited into this study. Participants were divided into two subgroups according to median admission omentin-1 concentration. $\delta 1$ was defined as (admission omentin-1 level) - (serum omentin-1 at 24 hours after admission) and $\delta 2$ was defined as (admission omentin-1 level) - (serum omentin-1 at 72 hours after admission). The change in left ventricular ejection fraction (LVEF) was regarded as (LVEF at 3 months post-STEMI) -(LVEF at 2 days post-STEMI).

Results: Admission omentin-1 level was the highest, while omentin-1 decreased over the following 3 days. The high admission omentin-1 group had lower peak muscle brain fraction of creatine kinase (CK-MB). Additionally, the change in LVEF and the global LVEF at 3 months post-STEMI all ameliorated significantly in the high admission omentin-1 group. For the time-dependent change in omentin-1, there were negative associations among $\delta 1, \delta 2$, and peak CK-MB. $\delta 1$ and $\delta 2$ also correlated positively with LVEF at 3 months post-STEMI. Most importantly, $\delta 1(r=0.346, p=0.012)$ and $\delta 2(r=0.439, p=0.001)$ also correlated positively with the change in LVEF. After multivariate linear regression analysis, $\delta 1$ (Beta $=0.026,95 \% \mathrm{CI} 0.011$ to $0.041, p=0.001$ ) and $\delta 2($ Beta $=0.024,95 \%$ CI 0.009 to $0.038, p=0.003)$ also remained associated with the change in LVEF.

Conclusions: The admission omentin-1 and time-dependent change in omentin-1 level all have a significant correlation with the early improvement of post-infarction myocardial function. While only the time-dependent change in omentin-1 ( $\delta 1$ and $\delta 2)$ remained associated with the early improvement of post-infarction myocardial function after multivariate linear regression analysis. The present research indicated that omentin-1 represents a promising adipocytokine to retard negative cardiac remodeling after STEMI.

\section{Key words: Omentin-1, STEMI, LVEF, CK-MB}

Abbreviations: STEMI: ST-segment elevation myocardial infarction; I/R: ischemia/reperfusion; CAD: coronary artery disease; ACS: acute coronary syndrome; PCI: percutaneous coronary intervention; LAD: left anterior descending coronary artery; BMI: body mass index; TIMI: thrombolysis in Myocardial infarction; TNF- $\alpha$ : tumour necrosis factor- $\alpha$; IL-6: interleukin-6; ELISA: enzyme-linked immunosorbent assay; CK-MB: muscle brain fraction of creatine kinase; NT-proBNP: N-terminal pro B-type natriuretic peptide; LVEF: left ventricular ejection fraction; AMI: acute myocardial infarction.

Address for correspondence: Yingxin Zhao, Department of Cardiology, Beijing Anzhen Hospital, Capital Medical University; Beijing Institute of Heart Lung and Blood Vessel Disease. \#2, Anzhenlu, ChaoyangDistrict, Beijing 100029, China E-mail: zyingxinmi@163.com

Received: September 24, 2018 Accepted for publication: January 8, 2019 


\section{Introduction}

Early reperfusion strategies and adjuvant pharmacological therapies limit myocardial injury and improve survival after ST-segment elevation myocardial infarction (STEMI) ${ }^{1,2}$. However, reperfusion itself can induce additional damage to ischemic myocardium termed myocardial ischemia/reperfusion (I/R) injury, which also contributes to negative post-infarction myocardial remodeling and subsequent heart dysfunction ${ }^{3,4)}$. Recently, clinical and experimental studies have indicated that the novel adipocytokine omentin-1 plays an important role in regulating I/R injury ${ }^{5)}$.

Omentin-1, also named intelectin-1, is a novel adipocytokine with 313 amino acids, which is predominantly secreted by the human visceral adipose tissue rather than subcutaneous adipose tissue ${ }^{6,7)}$. Serum omentin-1 declines significantly in patients with obesity and obesity-associated disorders, including impaired glucose regulation, type 2 diabetes mellitus, and metabolic syndrome ${ }^{7-9)}$. Serum levels of omentin-1 are also downregulated in patients with coronary artery disease $(\mathrm{CAD})^{10,11)}$, and upregulated in patients with the acute coronary syndrome (ACS) ${ }^{12,13)}$. Additionally, serum omentin-1 not only suppresses the process of atherosclerotic lesions ${ }^{13}$, 14) but also may represent a novel biomarker inversely correlated with plaque instability ${ }^{15)}$. Furthermore, serum omentin- 1 also ameliorates myocardial damage and myocardial function after successful reperfusion in patients with STEMI ${ }^{5}$.

However, there is no previous research concerning the kinetic changes in omentin-1 during the acutephase of STEMI and the influence of it on myocardial damage and function after reperfusion. Therefore, the main purpose of this study was to address the correlations among the admission omentin-1, time-dependent kinetic changes in omentin-1, and myocardial function in patients with STEMI after successful primary percutaneous coronary intervention (PCI).

\section{Methods}

\section{Subjects and Study Design}

Between June 2017 and May 2018, patients with anterior STEMI were recruited from two hospitals in Beijing, China. Inclusion criteria were age $>18$ years, typical symptoms $>30$ minutes and $<12$ hours, electrocardiogram sign (ST-segment elevation $>2 \mathrm{~mm}$ in at least two contiguous precordial leads or $>1 \mathrm{~mm}$ in at least two contiguous limb leads), the infarct-related artery being the left anterior descending (LAD) artery and $<70 \%$ stenosis of the non-infarct-related artery treated successfully by primary PCI. The success of primary PCI was defined as the recanalization of the LAD with $<30 \%$ residual stenosis of the infarct-related lesion. Patients with a previous history of myocardial infarction and PCI were excluded. In addition, we also excluded the patients with a serious concomitant disease, including acute infection, malignancy, liver and renal impairment, chronic inflammatory disease and overt heart failure (Killip class III or IV). Finally, a total of 52 patients were recruited into our study. Additionally, the patients were divided into two subgroups according to the median value of admission omentin-1 level. The study was approved by the regional Ethics Committee and conducted in compliance with the Declaration of Helsinki. In addition, we obtained the written informed consent from all the patients before enrollment.

\section{Treatment and Clinical Evaluation}

Doses of clopidogrel $600 \mathrm{mg}$, aspirin $300 \mathrm{mg}$ and intravenous heparin $(70-100 \mathrm{U} / \mathrm{kg})$ were administered before primary PCI. Primary PCI was conducted by experienced interventionists in accordance with current practice guidelines and was carried out mainly through the radial artery. In addition, the patients with a high thrombus burden were recommended treatment with thrombus aspiration. The choice of stent type (new generation drug-eluting stents) and adjunctive pharmacologic treatment were placed at the discretion of the operator.

Demographic data, such as age, height, body weight, body mass index (BMI), blood pressure, and Killip class were all obtained upon admission. Medical history of smoking, diabetes, and hypertension were also recorded. In addition to total ischemic time, we also recorded single/multivessel disease, angiographic thrombolysis in myocardial infarction (TIMI) flow grade pre-operation and post-operation, the number of stents, as well as medications used at discharge.

BMI was obtained via weight in kilograms divided by the square of height in meters. From symptom onset to recanalization of infarction-associated artery was defined as total ischemic time. Multivessel disease was defined as $>50 \%$ stenosis in at least one non-infarctrelated artery. Killip class on admission and angiographic data were all at the discretion of two experienced cardiologists.

\section{Blood Sampling and Biochemical Analysis}

The venous blood samples were collected with serum separation hoses (Becton-Dickinson) on admission, at 24 hours, and at 72 hours after admission, followed by centrifuging for 15 minutes at $2500 \mathrm{~g}$ and storage of serum samples at $-80^{\circ} \mathrm{C}$. The aim of collecting these blood samples was to assay omentin- 1 and two key inflammatory markers: tumor necrosis factor- $\alpha$ 
Table 1. Baseline characteristics

\begin{tabular}{|c|c|c|c|c|}
\hline & Total & Low omentin-1 group & High omentin-1 group & $p$-value \\
\hline Number & 52 & 26 & 26 & \\
\hline Age & $55.12 \pm 12.43$ & $56.50 \pm 13.89$ & $53.73 \pm 10.88$ & 0.427 \\
\hline Male gender (\%) & $40(76.9)$ & $17(65.4)$ & $23(88.5)$ & 0.097 \\
\hline Systolic BP (mmHg) & $120.46 \pm 16.53$ & $117.00 \pm 15.63$ & $123.92 \pm 16.97$ & 0.132 \\
\hline Diastolic BP (mmHg) & $77.54 \pm 12.39$ & $75.50 \pm 11.25$ & $79.58 \pm 13.33$ & 0.239 \\
\hline Body mass index $\left(\mathrm{kg} / \mathrm{m}^{2}\right)$ & $26.43 \pm 3.49$ & $27.24 \pm 3.55$ & $25.62 \pm 3.30$ & 0.094 \\
\hline Hypertension (\%) & $27(51.9)$ & $14(53.8)$ & $13(50.0)$ & 1.000 \\
\hline Diabetes (\%) & $10(19.2)$ & 7 (26.9) & $3(11.5)$ & 0.291 \\
\hline Current Smoking (\%) & $38(73.1)$ & $19(73.1)$ & $19(73.1)$ & 1.000 \\
\hline Pre-infarction angina (\%) & $15(28.8)$ & $9(34.6)$ & $6(23.1)$ & 0.541 \\
\hline Total ischemia time (min) & $359.88 \pm 206.47$ & $374.46 \pm 198.90$ & $345.31 \pm 216.71$ & 0.616 \\
\hline Killip class II on admission (\%) & $26(50.0)$ & $14(53.8)$ & $12(46.2)$ & 0.782 \\
\hline LVEF at 2 days post-STEMI (\%) & $55.29 \pm 7.26$ & $56.42 \pm 6.71$ & $54.15 \pm 7.73$ & 0.264 \\
\hline \multicolumn{5}{|l|}{ Laboratory examination } \\
\hline White blood cells $\left(10^{9} / \mathrm{L}\right)$ & $11.28 \pm 3.35$ & $11.86 \pm 3.83$ & $10.70 \pm 2.74$ & 0.212 \\
\hline Hemoglobin $(\mathrm{g} / \mathrm{L})$ & $142.48 \pm 18.33$ & $139.69 \pm 19.23$ & $145.27 \pm 17.29$ & 0.277 \\
\hline Platelet $\left(10^{9} / \mathrm{L}\right)$ & $238.31 \pm 55.55$ & $236.96 \pm 59.53$ & $239.65 \pm 52.41$ & 0.863 \\
\hline Fasting blood glucose (mmol/L) & $6.81 \pm 1.98$ & $7.20 \pm 2.14$ & $6.41 \pm 1.76$ & 0.150 \\
\hline Triglycerides (mmol/L) & $1.68 \pm 1.05$ & $1.94 \pm 1.25$ & $1.42 \pm 0.76$ & 0.079 \\
\hline LDL-C (mmol/L) & $2.72 \pm 0.92$ & $2.68 \pm 0.84$ & $2.76 \pm 0.76$ & 0.724 \\
\hline HDL-C (mmol/L) & $0.99 \pm 0.16$ & $0.96 \pm 0.15$ & $1.02 \pm 0.17$ & 0.199 \\
\hline Uric acid (mg/dl) & $328.90 \pm 85.43$ & $333.00 \pm 94.85$ & $324.81 \pm 76.52$ & 0.733 \\
\hline Serum creatinine (umol/L) & $59.04 \pm 14.56$ & $58.38 \pm 15.68$ & $59.69 \pm 13.62$ & 0.750 \\
\hline Peak CK-MB (U/L) & $217.82 \pm 141.81$ & $257.58 \pm 147.75$ & $178.05 \pm 126.12$ & 0.042 \\
\hline NT-proBNP (pg/ml) & $494.50(249.25,1610.00)$ & $1040.00(409.75,1940.75)$ & $426.00(206.50,918.25)$ & 0.008 \\
\hline \multicolumn{5}{|l|}{ Angiographic data (\%) } \\
\hline Infarct-related artery (LAD) & $52(100.0)$ & $26(100.0)$ & $26(100.0)$ & 1.000 \\
\hline Multivessel disease & $17(32.7)$ & $9(34.6)$ & $8(20.8)$ & 1.000 \\
\hline Gensini Score & $58.38 \pm 20.97$ & $61.62 \pm 18.55$ & $55.15 \pm 23.04$ & 0.271 \\
\hline Thrombus aspiration & $3(5.8)$ & $1(3.8)$ & $2(7.6)$ & 1.000 \\
\hline Tirofiban & $29(55.8)$ & $16(61.5)$ & $13(50.0)$ & 0.577 \\
\hline Stent implantation & $52(100.0)$ & $26(100.0)$ & $26(100.0)$ & 1.000 \\
\hline The number of stent $=1$ & $46(88.5)$ & $22(84.6)$ & $24(92.3)$ & 0.668 \\
\hline $\mathrm{TIMI}=0$, before PCI & $42(80.8)$ & $23(88.5)$ & $19(73.1)$ & 0.291 \\
\hline TIMI $<3$, after PCI & $14(26.9)$ & $10(38.5)$ & $4(15.4)$ & 0.116 \\
\hline \multicolumn{5}{|l|}{ Medication, before AMI (\%) } \\
\hline Aspirin & $4(7.7)$ & $3(11.5)$ & $1(3.8)$ & 0.610 \\
\hline Statin & $8(15.4)$ & $3(11.5)$ & $5(19.2)$ & 0.703 \\
\hline ACEI/ARB & $8(15.4)$ & $5(19.2)$ & $3(11.5)$ & 0.703 \\
\hline Beta-block & $2(3.8)$ & $2(7.7)$ & $0(0.0)$ & 0.490 \\
\hline Metformin & $6(11.5)$ & $5(19.2)$ & $1(3.8)$ & 0.191 \\
\hline Other hypoglycemic agents & $2(3.8)$ & $2(7.7)$ & $0(0.0)$ & 0.490 \\
\hline Insulin & $1(1.9)$ & $0(0.0)$ & $1(3.8)$ & 1.000 \\
\hline \multicolumn{5}{|l|}{ Medication, discharge (\%) } \\
\hline Aspirin & $52(100.0)$ & $26(100.0)$ & $26(100.0)$ & 1.000 \\
\hline Clopidogrel & $52(100.0)$ & $26(100.0)$ & $26(100.0)$ & 1.000 \\
\hline
\end{tabular}


(Cont. Table 1)

\begin{tabular}{|c|c|c|c|c|}
\hline & Total & Low omentin-1 group & High omentin-1 group & $p$-value \\
\hline \multicolumn{5}{|l|}{ Medication, discharge (\%) } \\
\hline Statin & $52(100.0)$ & $26(100.0)$ & $26(100.0)$ & 1.000 \\
\hline ACEI/ARB & $43(82.7)$ & $22(84.6)$ & $21(80.8)$ & 1.000 \\
\hline Beta-block & $50(96.2)$ & $25(96.2)$ & $25(96.2)$ & 1.000 \\
\hline Metformin & $7(13.5)$ & $6(23.1)$ & $1(3.8)$ & 0.099 \\
\hline Other hypoglycemic agents & $2(3.8)$ & $2(7.7)$ & $0(0.0)$ & 0.490 \\
\hline Insulin & $1(1.9)$ & $0(0.0)$ & $1(3.8)$ & 1.000 \\
\hline
\end{tabular}

Data are presented as Mean \pm SD, median (low quartile ,upper quartile), or number (\%)

BP blood pressure, LVEF left ventricular ejection fraction, LDL-C low-density lipoprotein cholesterol, HDL-C high-density lipoprotein cholesterol, CK-MB muscle brain fraction of creatine kinase, NT-proBNP N-terminal pro B-type natriuretic peptide, LAD left anterior descending coronary artery, TIMI thrombolysis in myocardial infarction, PCI percutaneous coronary intervention, AMI acute myocardial infarction, ACEI/ARB angiotensin-converting enzyme inhibitor/angiotensin receptor blocker

(TNF- $\alpha$ ) and interleukin-6 (IL-6). The concentrations of serum omentin-1 and inflammatory cytokines were measured in duplicate by commercially available enzymelinked immunosorbent assay (ELISA) kits according to the manufacturer's instructions (BLUE GENE, Shanghai, China). Both ELISA intra-assay and inter-assay coefficients of variation in our study were $<5 \%$. Finally, we defined (admission omentin-1 level) - (serum omentin- 1 at 24 hours after admission) as $\delta 1$ and (admission omentin-1 level) - (serum omentin-1 at 72 hours after admission) as $\delta 2$.

Peak MB fraction of creatine kinase $(\mathrm{CK}-\mathrm{MB})$ correlated well with infarct size and prognosis after acute myocardial infarction ${ }^{16)}$. Thus, CK-MB levels were measured on admission, the following evening, and morning to identify peak CK-MB. N-terminal proB-type natriuretic peptide (NT-proBNP) measured 2 to 4 days following STEMI independently forecast myocardial function and 2-year survival, which is superior to BNP and other cardiac peptides ${ }^{17,18)}$. So, NTproBNP at day 3 post-STEMI was determined. The rest of the routine biochemical parameters were also determined in the Central Laboratory of Beijing Anzhen Hospital.

\section{Evaluation of Myocardial Function with Echocar- diography}

Doppler echocardiography was performed by the same two experienced doctors at 2 days and 3 months post-STEMI. Standard echocardiography views were obtained with the supervision of experienced cardiologists. Left ventricular ejection fraction (LVEF) was acquired with the modified Simpson biplane method. The change in LVEF was regarded as (LVEF at 3 months post-STEMI) - (LVEF at 2 days post-STEMI).

\section{Statistical Analysis}

Normality distribution of continuous variables was assessed by Kolmogorov-Smirnov test. Continuous data are expressed as the mean \pm standard deviation or median value with interquartile range. Mean values of the data were compared by independent-sample $t$-tests but median values of variables were compared by the MannWhitney $U$ test. Categorical variables are presented as percentages and were analyzed by chi-squared tests. Oneway analysis of variance was used to test the trend of time course of serum omentin-1. The association between variables was assessed by Pearson correlation coefficients. In addition, the associations between variables and LVEF change from baseline to 3 months were determined by univariate analysis and multivariate linear regression analysis. All the statistical analyzes were performed using SPSS 20.0 software (SPSS Inc., Chicago, IL, USA). $P$ values $<0.05$ were considered statistically significant.

\section{Results}

\section{Baseline Characteristics}

The baseline characteristics of patients recruited into this study are presented in Table 1 . The mean age of the recruited patients was $55.12 \pm 12.43$ years old, and $76.9 \%$ were male. The prevalence of current smoker, hypertension, and diabetes were $73.1 \%, 51.9 \%$, and $19.2 \%$, respectively. In addition, peak $\mathrm{CK}-\mathrm{MB}$ was $217.82 \pm 141.8 \mathrm{U} / \mathrm{L}$ and the median NT-proBNP at day 3 post-STEMI was $494.50(249.25,1610.00) \mathrm{pg} /$ $\mathrm{ml}$. Furthermore, $67.3 \%$ of the recruited patients had single vessel disease. Most importantly, the mean total ischemic time was $359.88 \pm 206.47$ minutes, which revealed that all the patients recruited obtained timely reperfusion. We also divided the patients into two subgroups. Except for peak CK-MB, NT-proBNP, and 
change in LVEF from baseline to 3 months, no significant differences were observed between the two subgroups.

\section{Change in Serum Omentin-1 in Acute STEMI}

The sequential change in serum omentin-1 concentration in the recruited patients was tested first. The mean concentration of serum omentin- 1 on admission, at 24 hours, and at 72 hours after admission was $934.75 \pm 249.82 \mathrm{pg} / \mathrm{ml}, 833.87 \pm 197.27 \mathrm{pg} / \mathrm{ml}$ and $844.80 \pm 220.80 \mathrm{pg} / \mathrm{ml}$, respectively $(p$ trend $=0.044)$. As indicated in Fig. 1A, admission omentin-1 was the highest, while omentin-1 declined rapidly the following 72 hours. Additionally, as shown in Fig. 1B and Fig. 1C, baseline serum omentin-1 positively correlated with $\delta 1$ $(r=0.626, p<0.001)$ and $\delta 2(r=0.473, p<0.001)$.

Serum Omentin-1 and Inflammatory Cytokine Analysis

Correlation between serum omentin-1, time-dependent change in omentin-1, and inflammatory markers are presented in Table 2. For admission omentin-1 with inflammatory cytokines, admission omentin-1 not only correlated positively with admission IL-6 $(r=0.786$, $p<0.001)$ and admission TNF- $\alpha(p=0.565, p<0.001)$ but also correlated positively with IL- 6 and TNF- $\alpha$ at other time points. In addition, omentin-1 concentration at 24 and 72 hours after admission also had consistent correlation with inflammatory cytokines at each time point. Furthermore, there were consistent correlations between $\delta 1$ and IL- 6 on admission $(r=0.519$, $p<0.001)$, at 24 hours $(r=0.421, p=0.002)$, and at 72 hours $(r=0.489, p<0.001)$ after admission. $\delta 1$ also correlated with admission TNF- $\alpha(r=0.362, p=0.008)$ and TNF- $\alpha$ at 72 hours $(r=0.275, p=0.048)$ after admission. For $\delta 2$, there were also positive correlations with IL-6 on admission $(r=0.294, p=0.034)$ and at 24 hours $(r=0.297, p=0.033)$ after admission.

Association between Omentin-1 and Peak CK-MB with NT-proBNP

As shown in Table 1, compared with the low admission omentin-1 group, the high admission omentin-1 subgroup had a lower level peak CK-MB (178.05 \pm 126.12 U/L vs. $257.58 \pm 147.75 \mathrm{U} / \mathrm{L}, p=0.042)$. As shown in Fig. 2A and Fig. 2B, there were inverse correlations between $\delta 1 \quad(r=-0.280, p=0.044), \delta 2(r=$ $-0.294, p=0.034)$ and peak CK-MB. Additionally, as presented in Table 1, the patients in the subgroup with high admission omentin-1 also exhibited a lower NTproBNP level [426.00 $(206.50,918.25) \mathrm{pg} / \mathrm{ml}$ vs. 1040.00 $(409.75,1940.75) \mathrm{pg} / \mathrm{ml}, p=0.008]$ measured at 3 days post-STEMI. As revealed in Fig. 2C and Fig. 2D, there were also negative correlations between $\delta 1(r=-0.384$,
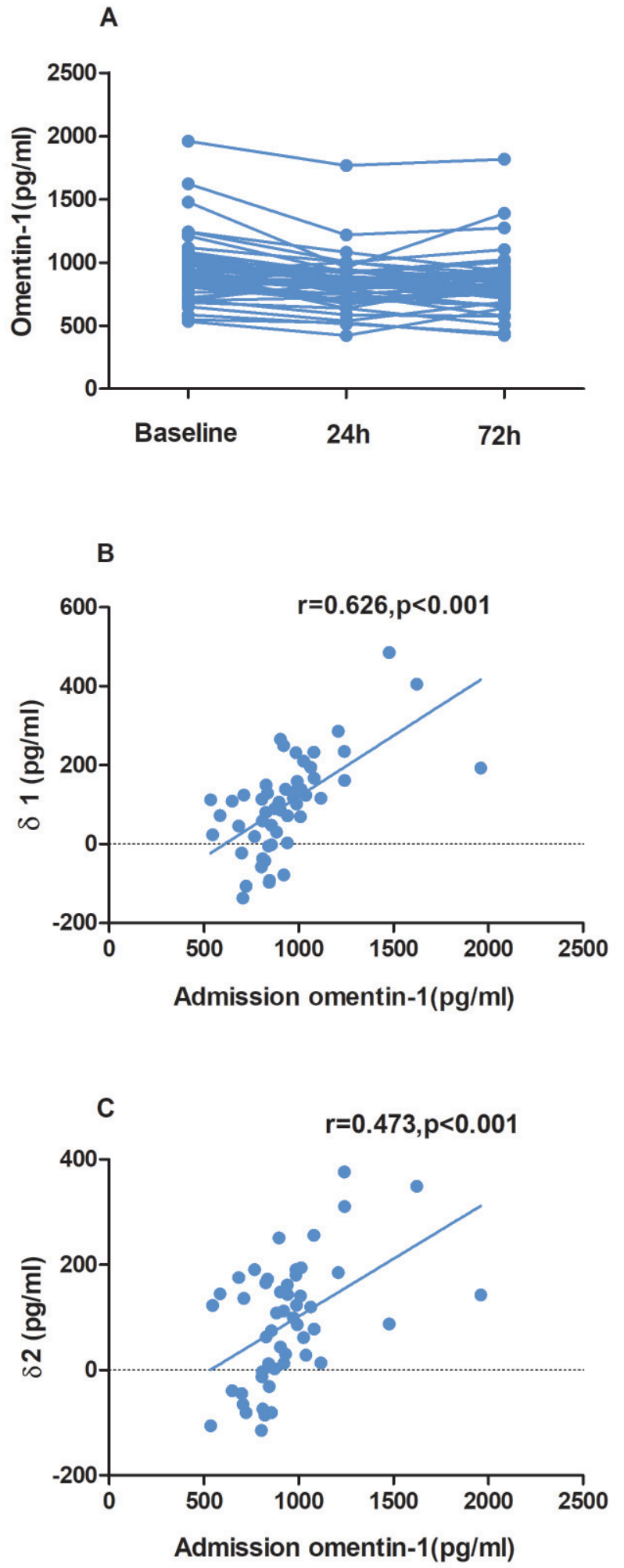

Fig. 1. The sequential change in serum omentin-1 concentration (A). The association between admission omentin- 1 and $\delta 1(\mathrm{~B})$ as well as $\delta 2(\mathrm{C})$. (Admission omentin-1 level) - (serum omentin-1 at 24 hours after admission) as $\delta 1$ and (admission omentin-1 level) - (serum omentin-1 at 72 hours after admission) as $\delta 2$. 
Table 2. Correlation between serum omentin-1, time-dependent change of omentin-1 and inflammatory markers

\begin{tabular}{|c|c|c|c|c|c|}
\hline & $\begin{array}{c}\text { Baseline } \\
\text { omentin-1 }\end{array}$ & $\begin{array}{l}\text { Omentin-1 } \\
(24 \mathrm{~h})\end{array}$ & $\begin{array}{l}\text { Omentin-1 } \\
\quad(72 \mathrm{~h})\end{array}$ & $\delta 1$ & $\delta 2$ \\
\hline Admission IL-6 & $0.786(<0.001)$ & $0.664(<0.001)$ & $0.741(<0.001)$ & $0.519(<0.001)$ & $0.294(0.034)$ \\
\hline IL-6 (72 h) & $0.797(<0.001)$ & $0.698(<0.001)$ & $0.772(<0.001)$ & $0.489(<0.001)$ & $0.265(0.058)$ \\
\hline Admission TNF- $\alpha$ & $0.565(<0.001)$ & $0.483(<0.001)$ & $0.509(<0.001)$ & $0.362(0.008)$ & $0.257(0.066)$ \\
\hline
\end{tabular}

IL-6 interleukin-6, TNF- $\alpha$ tumor necrosis factor-alpha, $\delta 1$ as (admission omentin-1 level) - (omentin-1 level at 24 hour after admission), $\delta 2$ as (admission omentin-1 level) - (omentin-1 level at 72 hour after admission).

$p=0.005), \delta 2(r=-0.420, p=0.002)$ and NT-proBNP measured at 3 days post-STEMI.

\section{Correlation between Omentin-1 and the Early Change in Myocardial Function}

After calculating the change in LVEF from baseline to 3 months post-STEMI, as shown in Fig. 3A, our data demonstrated that the absolute change in LVEF improved significantly in the high admission omentin-1 group compared to the low admission omentin-1 group $(5.27 \% \pm 7.11 \%$ vs. $-0.46 \% \pm 7.84 \%, P=0.008)$. Additionally, as indicated in Fig. 3B, the global LVEF in the high admission omentin-1 group improved significantly $(59.41 \% \pm 7.23 \%$ vs. $54.15 \% \pm 7.73 \%, p=0.014)$, but the low admission omentin-1 group was without such improvement $(55.96 \% \pm 8.84 \%$ vs. $56.42 \% \pm 6.71 \%$, $p=0.833$ ).

As for the time-dependent change in serum omentin-1 with LVEF at 3 months post-STEMI, there were moderate correlations between $\delta 1(r=0.392, p=0.004)$, $\delta 2(r=0.382, p=0.005)$, and LVEF at 3 months postSTEMI (Fig. 4A, Fig.4B). Most importantly, moderate but high associations were also observed between $\delta 1(r=0.346, p=0.012), \delta 2(r=0.439, p=0.001)$, and change in LVEF from baseline to 3 months post-STEMI (Fig.4C, Fig.4D). Last but not the least, in multivariate linear regression model, which included age, sex, and other variables with $p<0.05$ in univariate analysis as shown in Table 3, $\delta 1$ (Beta $=0.026$, 95\% CI 0.011 to $0.041, p=0.001)$ remained associated with the absolute change in LVEF from baseline to 3 months postSTEMI in multiple linear regression model A. Additionally, in model $\mathrm{B}, \delta 2$ (Beta $=0.024,95 \%$ CI 0.009 to $0.038, p=0.003$ ) also correlated with the change in LVEF from baseline to 3 months post-STEMI.

\section{Discussion}

This is the first study to manifest time-dependent kinetic changes in omentin-1 levels associated with the early improvement of myocardial function in patients with their first anterior STEMI after primary PCI. In the present study, we demonstrated several relationships. First, serum omentin-1 was the highest on admission but declined significantly in the following 72 hours. Additionally, admission omentin-1 level correlated positively with time-dependent kinetic changes in omentin-1 level ( $\delta 1$ and $\delta 2$ ). Second, peak $\mathrm{CK}-\mathrm{MB}$ and NT-proBNP at 72 hours post-STEMI were significantly lower in the high admission omentin-1 subgroup. Additionally, the high admission omentin-1 group improved markedly in the change in LVEF compared to the low omentin-1 group. Third, time-dependent kinetic changes in omentin-1 level ( $\delta 1$ and $\delta 2)$ had negative correlations with peak $\mathrm{CK}-\mathrm{MB}$ and NT-proBNP at 72 hours post-STEMI. Furthermore, the $\delta 1$ and $\delta 2$ not only correlated positively with LVEF at 3 months postSTEMI but also correlated with the change in LVEF.

Accumulating evidence indicated omentin-1, as a novel adipocytokine with cardioprotective effects, is downregulated in people with atherosclerosis and risk factors associated with atherosclerosis, including obesity, diabetes, hypertension, and dyslipidemia ${ }^{7,19,20)}$. Watanabe et al. and Du et al. further revealed that the concentration of omentin-1 was declined in the serum, epicardial adipose tissue and coronary endothelium in patients with $\mathrm{CAD}^{11,13)}$. However, the omentin-1 concentration increased markedly in the medial layer vascular smooth muscle and macrophage-derived foam cells within advanced plaques and circulating blood in patients with ACS ${ }^{13)}$. Saddic et al. and Kadoglou et al. further showed that serum omentin-1 increased significantly after acute myocardial ischemia ${ }^{12,21)}$. All these evidence suggests that serum omentin-1 not only was a passive risk factor for chronic CAD but was also an acute-phase reactant with cardioprotective effects during ACS. In addition, metformin may raise serum omentin-1 level in patients with acute myocardial infarction (AMI) and decrease the serum cardiac troponin concentration $^{22)}$. Weight loss, statin, and other hypoglyce- 
A

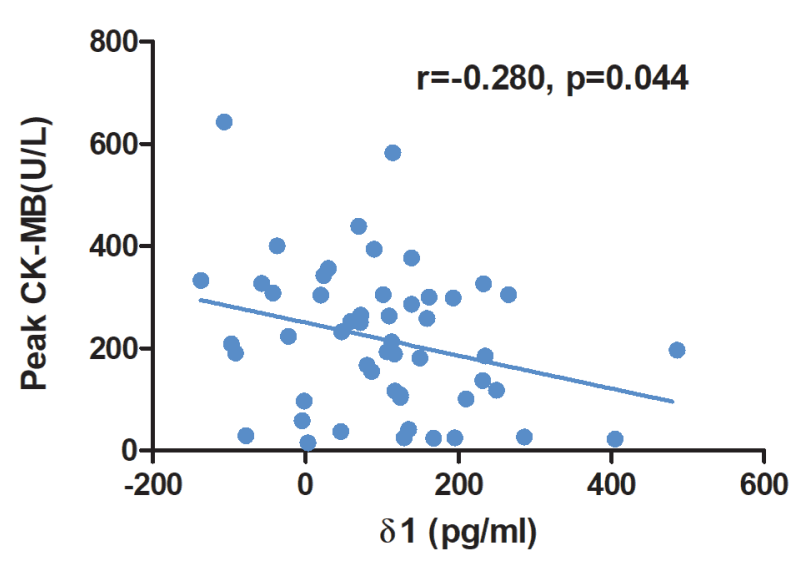

C

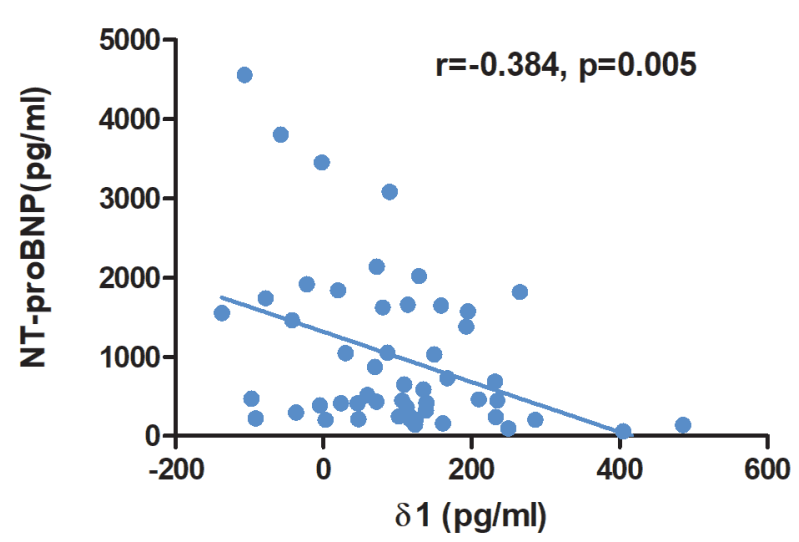

B

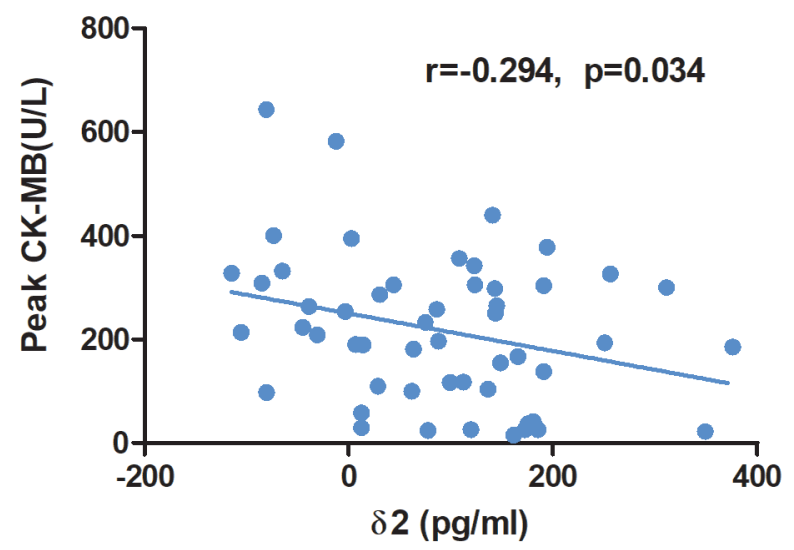

D

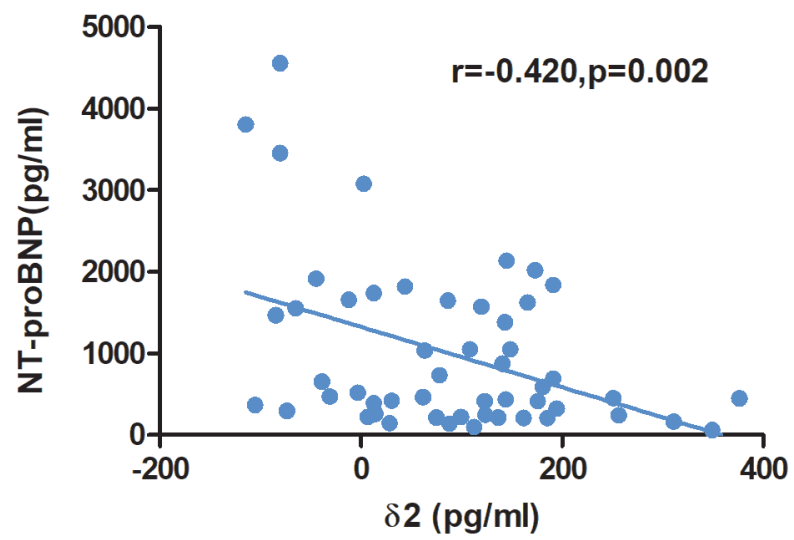

Fig. 2. orrelation between $\delta 1(\mathrm{~A}), \delta 2(\mathrm{~B})$ and peak CK-MB. Association between $\delta 1(\mathrm{C}), \delta 2(\mathrm{D})$ and NT-proBNP measured at 3 days post-STEMI. (Admission omentin-1 level) - (serum omentin-1 at 24 hours after admission) as $\delta 1$ and (admission omentin-1 level) - (serum omentin-1 at 72 hours after admission) as $\delta 2$; CK-MB, muscle brain fraction of creatine kinase; NTproBNP, N-terminal pro B-type natriuretic peptide; STEMI, ST-segment elevation myocardial infarction

mic agents, such as liraglutide and exenatide, may also increase serum omentin-1 level ${ }^{23-26)}$, but the evidence in patients with AMI was limited.

As we all know, myocardial I/R injury is characterized by an acute level of high-grade inflammatory response with rapid formation and release of TNF- $\alpha$ and IL- $6^{27,28)}$. In our present study, anti-inflammatory mediator omentin-1 correlated positively with the two key cytokines, TNF- $\alpha$ and IL-6, during the acute-phase of STEMI. Therefore, our study further confirmed that omentin-1 may act as an acute-phase reactant to counteract the inflammatory response for the sake of cardiovascular protection during the process of myocardial I/R.

Now, accumulating evidence has indicated that baseline omentin-1 increased significantly in circulat- ing blood in patients with $A C S^{12,13,21)}$. Our present study extends prior findings by indicating that increased admission omentin-1 declined transiently during the acute-phase of STEMI. The accumulation of omentin-1 in injured cardiovascular tissues as suggested by in vivo and in vitro experiments may ${ }^{13)}$, at least in part, account for the post-procedural transient decrement of serum omentin-1. Additionally, in patients with STEMI, the baseline adiponectin also decreased transiently, maybe due to the accumulation of adiponectin in injured cardiovascular tissues mediated by T-cadherin ${ }^{29-32)}$. Thus, this study calls for a future study to determine the mediators that play a critical role in cardiovascular tissue accumulation of omentin-1.

Kataoka et al. showed that high levels of circulating omentin-1 in mice limited myocardial injury and 
myocardial infarction size ${ }^{5)}$. In our present study, we have shown that in patients with STEMI, peak CK$\mathrm{MB}$ also declined significantly in the subgroup with a high level of admission omentin-1. Natsukawa et al. reported that the transient decrement of adiponectin from baseline to bottom during acute-phase STEMI was significantly associated with the area under the curve of $\mathrm{CK}-\mathrm{MB}^{30)}$. In our present study, both $\delta 1$ and $\delta 2$ also correlated negatively with peak CK-MB. Subsequently, we found the absolute change in LVEF from baseline to 3 months post-STEMI improved significantly in patients with a high level of baseline omentin-1. Similar to our finding, Kataoka et al. also confirmed that high levels of serum omentin-1 in post-STEMI patients were correlated with the improvement of myocardial function $^{5)}$. Most importantly, our study demonstrated that time-dependent changes in omentin- $1, \delta 1$, and $\delta 2$ correlated positively with the improvement of myocardial function for the first time. In addition, as Narumi et.al reported, high baseline omentin-1 level may have beneficial effects on cardiac prognosis in patients with heart failure or reduced $\mathrm{LVEF}^{33)}$, whereas the effects of timedependent change in omentin-1 in patients with heart failure were unknown.

Omentin-1 not only stimulated vasodilation of the isolated aorta via endothelium-dependent NO but also improved endothelial cell function and the process of revascularization in circumstances of acute ischemia via the AKt/eNOS pathway ${ }^{34,35)}$. For the antiinflammatory effect, omentin-1 stimulated the differentiation of macrophages into the M2 phenotype exerting an overwhelming anti-inflammatory action, rather than the M1 phenotype with proinflammatory action ${ }^{13)}$. In addition, omentin- 1 attenuated the vascular inflammatory response via various kinds of intracellular signaling pathways ${ }^{36-38)}$. Therefore, the anti-inflammatory and modulation of endothelial function features may account, at least in part, for omentin-1 being protective against I/R injury. In addition to myocardial infarction, other forms of cardiomyocyte death, including apoptosis, autophagy, and necroptosis were also responsible for the final infarct size and myocardial dysfunction in the post-STEMI patients ${ }^{3)}$. Omentin-1 can suppress the apoptosis of the myocyte associated with I/R via the mutually independent AMPK and Akt signal pathways, which was also responsible for the improvement in myocardial damage and function post-STEMI ${ }^{5}$. Whether omentin-1 has an influence on autophagy and necroptosis calls for further study. Furthermore, the cross talk between omentin-1 and other adipocytokines may also participate in regulating myocardial necrosis and subsequent myocardial remodeling, which needs further confirmation by large-scale clinical studies and in vitro experiments ${ }^{12,39)}$.
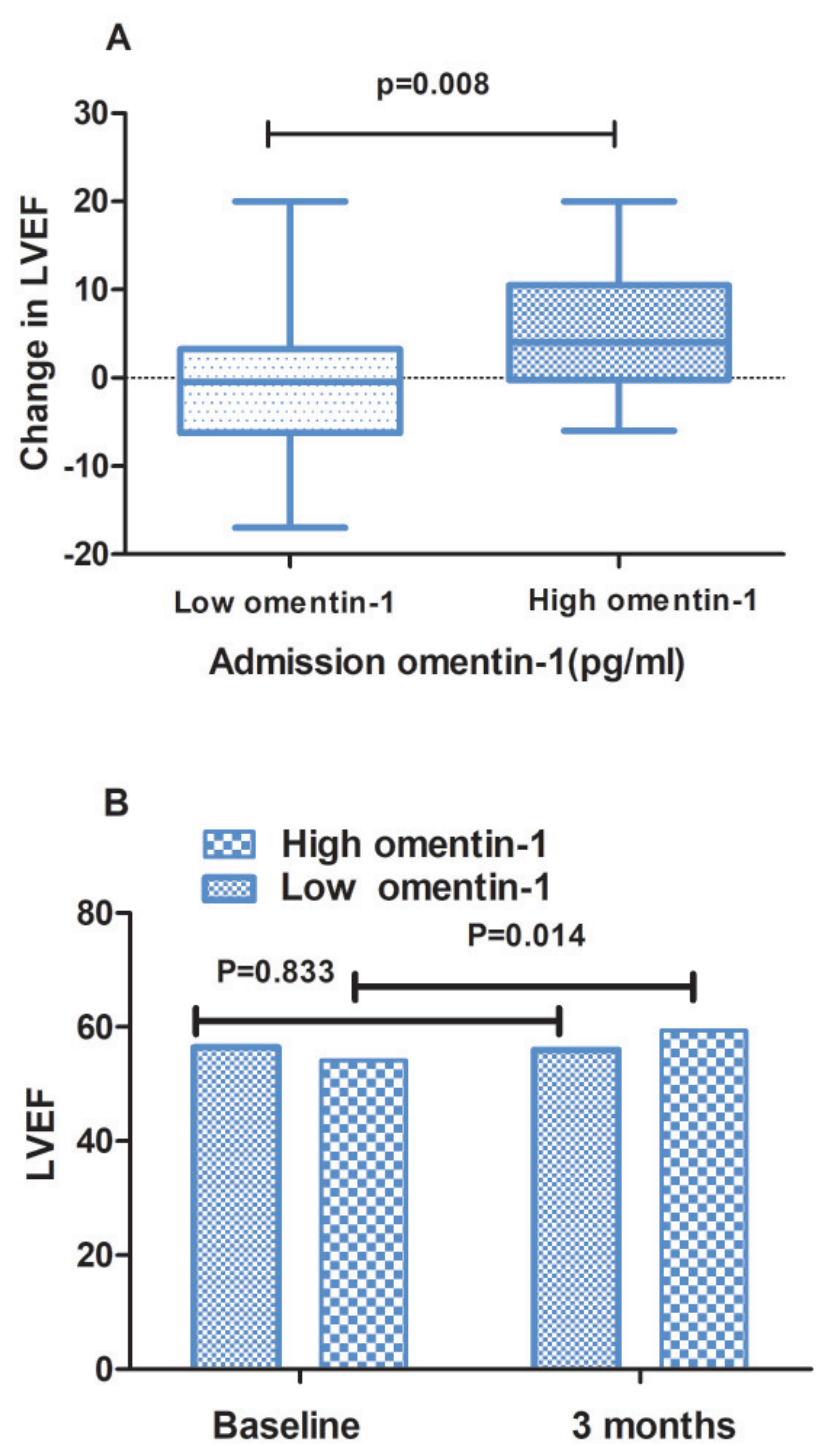

Fig. 3. The change in LVEF from baseline to 3 months in low and high admission omentin-1 group (A). The LVEF in patients with low and high omentin-1 group at baseline and 3 months post-STEMI (B). LVEF, left ventricular ejection fraction; STEMI, ST-segment elevation myocardial infarction.

Watanabe et al. showed through in vivo and in vitro experiments that almost all exogesly infused omentin-1 accumulated in the injured cardiovascular tissue, including the adventitia tissue of the aorta, myocardium and macrophages, without significant change in serum omentin-1 levels ${ }^{13)}$. In STEMI patients, examination of the accumulation of omentin-1 in the infarct region was impossible, but a transient decrement of serum omentin-1 in STEMI patients may imply the accumulation of omentin-1 in the infarct region. The 
A

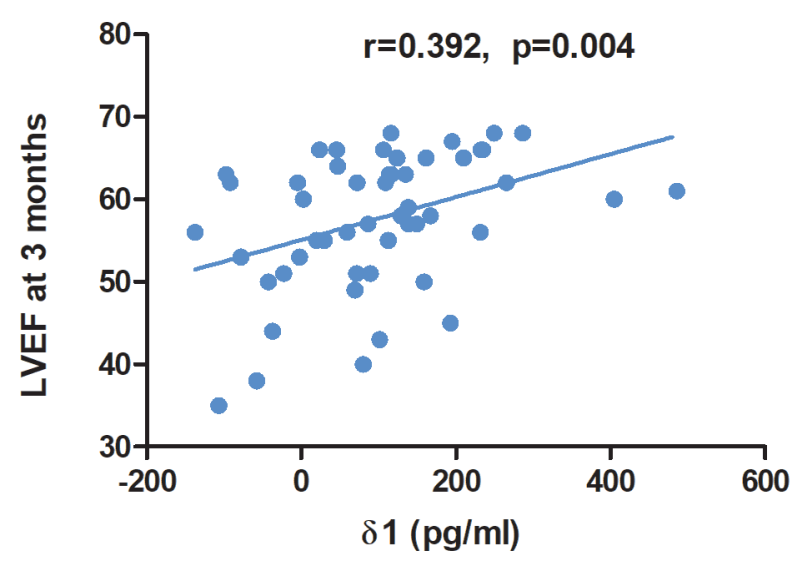

C

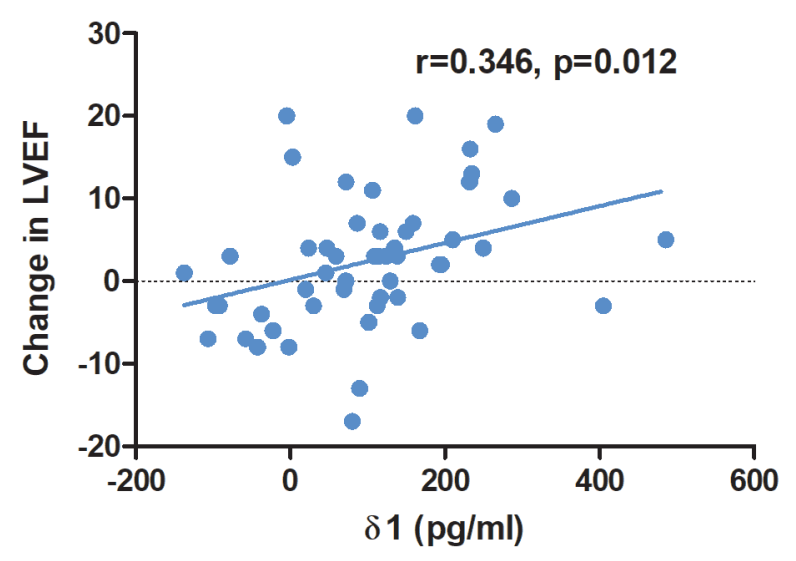

B

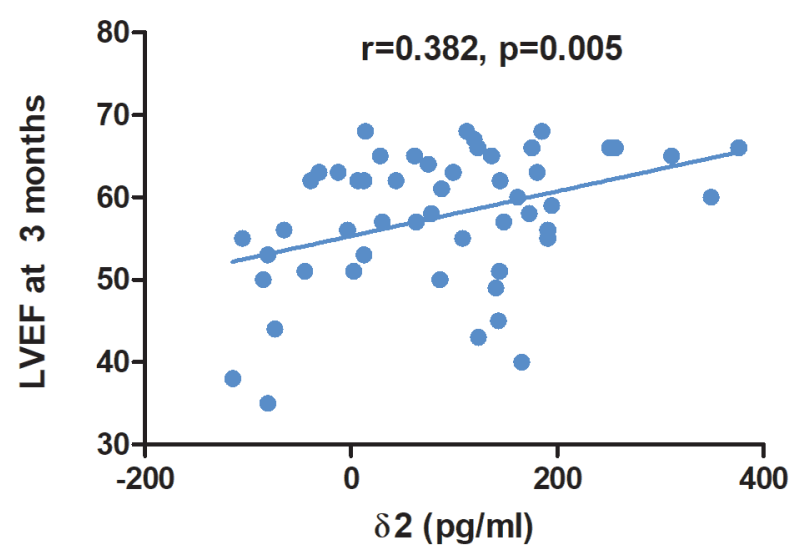

D

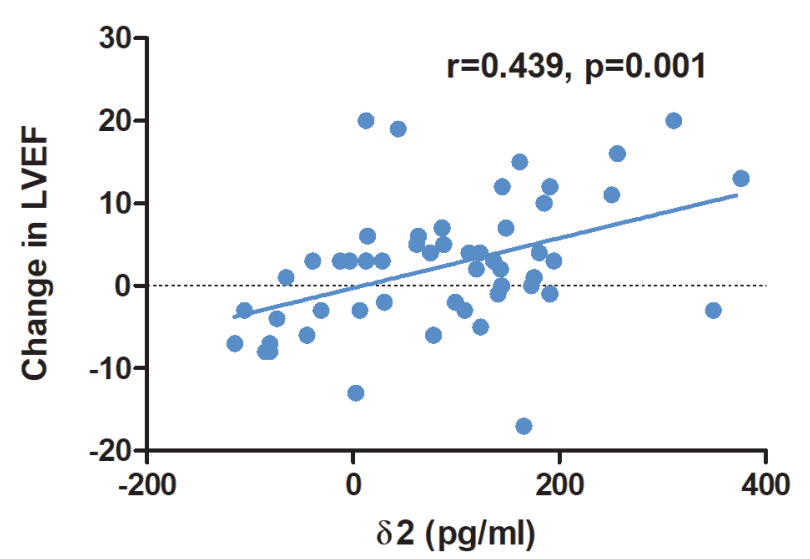

Fig.4. Correlation between $\delta 1(\mathrm{~A}), \delta 2(\mathrm{~B})$ and LVEF at 3 months after procedure. Correlation between $\delta 1(\mathrm{C}), \delta 2(\mathrm{D})$ and the change in LVEF. (Admission omentin-1 level) - (serum omentin-1 at 24 hours after admission) as $\delta 1$ and (admission omentin-1 level) - (serum omentin-1 at 72 hours after admission) as $\delta 2$; LVEF, left ventricular ejection fraction.

accumulation of omentin-1 in the infarct region may exert cardioprotective effects. As shown in Fig. 2 and Fig. 4, a transient decrement of serum omentin-1 $(\delta 1$ and $\delta 2$ ) not only had inverse correlation with peak $\mathrm{CK}-\mathrm{MB}$ and NT-proBNP at 72 hours post-STEMI, but also correlated positively with LVEF at 3 months post-STEMI and the early improvement in LVEF, suggesting the possibility that accumulation of omentin-1 may reduce myocardial necrosis and improve postinfarction myocardial function.

In our present study, admission omentin-1 and inflammatory cytokines (TNF- $\alpha$ and IL-6) all have positive effects on the cardiovascular tissue accumulation of omentin-1. If we can identify the mediators that play a critical role in cardiovascular tissue accumulation of omentin-1 during I/R injury, we may open up a novel therapeutic window for I/R injury.

\section{Study Limitations}

There are several limitations to this study. First, this was a cross-sectional study with a relatively small sample size. To further elucidate the relationship between time-dependent kinetic changes in omentin-1 level and the recovery of myocardial function large scale clinical studies are required. Second, we only enrolled patients with first anterior STEMI who underwent primary PCI, which may affect the outcome because we may have missed some critical STEMI patients. Third, the LVEF was only assessed by Doppler echocardiography, future studies should apply cardiac magnetic resonance imaging or single-photon emission computed 
Table 3. Correlation between absolute change of LVEF and variables using univariate and multivariate linear regression analysis

\begin{tabular}{|c|c|c|c|c|c|c|}
\hline Variable & \multicolumn{6}{|c|}{ Absolute change of LVEF from baseline to 3 months post-STEMI } \\
\hline Model A & Beta & $95 \% \mathrm{CI}$ & $P$-value & Beta & $95 \% \mathrm{CI}$ & $P$-value \\
\hline Age & -0.011 & -0.193 to 0.171 & 0.902 & -0.094 & -0.245 to 0.058 & 0.219 \\
\hline HDL & -14.919 & -28.418 to -1.420 & 0.031 & -15.448 & -26.684 to -4.211 & 0.008 \\
\hline Baseline LVEF & -0.465 & -0.747 to -0.183 & 0.002 & -0.488 & -0.732 to -0.243 & $<0.001$ \\
\hline$\delta 1$ & 0.022 & 0.005 to 0.040 & 0.012 & 0.026 & 0.011 to 0.041 & 0.001 \\
\hline Admission omentin-1 & 0.006 & -0.003 to 0.015 & 0.206 & & & \\
\hline Admission IL-6 & 0.033 & -0.024 to 0.089 & 0.256 & & & \\
\hline Mode B & Beta & $95 \% \mathrm{CI}$ & $p$-value & Beta & $95 \% \mathrm{CI}$ & $p$-value \\
\hline Age & -0.011 & -0.193 to 0.171 & 0.902 & -0.037 & -0.187 to 0.114 & 0.625 \\
\hline Sex & -6.808 & -11.756 to 1.861 & 0.008 & -4.509 & -8.934 to -0.085 & 0.046 \\
\hline FBG & -1.405 & -2.473 to -0.337 & 0.011 & -0.722 & -1.460 to 0.195 & 0.120 \\
\hline HDL & -14.919 & -28.418 to -1.420 & 0.031 & -7.606 & -18.283 to 3.070 & 0.158 \\
\hline Baseline LVEF & -0.465 & -0.747 to -0.183 & 0.002 & -0.444 & -0.692 to -0.196 & 0.001 \\
\hline$\delta 2$ & 0.030 & 0.013 to 0.048 & 0.001 & 0.024 & 0.009 to 0.038 & 0.003 \\
\hline Admission omentin-1 & 0.006 & -0.003 to 0.015 & 0.206 & & & \\
\hline Admission IL-6 & 0.033 & -0.024 to 0.089 & 0.256 & & & \\
\hline
\end{tabular}

LVEF left ventricular ejection fraction, CI confidence interval, FBG fasting blood-glucose, HDL high-density lipoprotein, $\delta 1$ as (omentin-1 leve1 on admission) - (omentin-1 level at 24 hours after admission), IL-6 interleukin-6, TNF- $\alpha$ tumor necrosis factor-alpha, $\delta 2$ as (admission omentin-1 level)-(omentin-1 level at 72 hours after admission).

tomography imaging to assess LVEF exactly and quantify the infarct size. Fourth, our research failed to determine whose omentin-1 levels might be lower before the onset of AMI, which needs further investigation by future research. Finally, after multiple linear regression analysis, $\delta 1$ and $\delta 2$ remained associated with the absolute change in LVEF from baseline to 3 months post-STEMI. However, the influence of residual confounding variables cannot be excluded.

\section{Conclusions}

In patients with their first anterior STEMI who underwent primary PCI, the early recovery of postinfarction myocardial function were significantly associated with admission omentin-1 levels and the timedependent change in serum omentin-1. Through multivariate linear regression analysis, only the time-dependent change in omentin-1 ( $\delta 1$ and $\delta 2)$ remained associated with the early improvement of post-infarction myocardial function. Our data suggested that omentin-1 is a novel adipocytokine that suppresses myocardial I/R injury and subsequent negative remodeling. Future studies are warranted to further elucidate the underlying mechanism between omentin-1 and myocardial I/R injury as well as subsequent negative remodeling. In addition, identifying the key mediators regulating cardiovascular tissue accumulation of omentin-1 is also very important.

\section{Acknowledgement}

We convey thanks to the following doctors, Meng Li, Kun Wang, Xue-Jie Wang, and Jie Sun for their help in collecting the blood sample. This work was supported by the Beijing Municipal science and Technology Commission (NO. Z171100000417042). 


\section{Conflict of Interest}

The authors declare that they have no competing interests.

\section{References}

1) Puymirat E, Simon T, Steg PG, Schiele F, Gueret P, Blanchard D, Khalife K, Goldstein P, Cattan S, Vaur L, Cambou JP, Ferrieres J, Danchin N: Association of changes in clinical characteristics and management with improvement in survival among patients with ST-elevation myocardial infarction. JAMA, 2012; 308: 998-1006

2) Gale CP, Allan V, Cattle BA, Hall AS, West RM, Timmis A, Gray HH, Deanfield J, Fox KAA, Feltbower R: Trends in hospital treatments, including revascularisation, following acute myocardial infarction, 2003-2010: a multilevel and relative survival analysis for the National Institute for Cardiovascular Outcomes Research (NICOR). Heart, 2014; 100: 582-589

3) Ibáñez B, Heusch G, Ovize M, Werf FVD: Evolving Therapies for Myocardial Ischemia/Reperfusion Injury. J Am Coll Cardiol, 2015; 65: 1454-1471

4) Montecucco F, Carbone F, Schindler TH: Pathophysiology of ST-segment elevation myocardial infarction: novel mechanisms and treatments. Eur Heart J, 2016; 37: 12681283

5) Kataoka Y, Shibata R, Ohashi K, Kambara T, Enomoto T, Uemura Y, Ogura Y, Yuasa D, Matsuo K, Nagata T, Oba T, Yasukawa H, Numaguchi Y, Sone T, Murohara T, Ouchi N: Omentin Prevents Myocardial Ischemic Injury Through AMP-Activated Protein Kinase- and AktDependent Mechanisms. J Am Coll Cardiol, 2014; 63: 2722-2733

6) Yang R, Lee M, Hu H, Pray J, Wu H, Hansen BC, Shuldiner AR, Fried SK, McLenithan JC, Gong D: Identification of omentin as a novel depot-specific adipokine in human adipose tissue: possible role in modulating insulin action. Am J Physiol-Endoc M, 2006; 290: E1253-E1261

7) Watanabe T, Watanabe-Kominato K, Takahashi Y, Kojima M, Watanabe R: Adipose Tissue-Derived Omentin-1 Function and Regulation. Compr Physiol, 2017; 7: 765

8) Cm DSB, Yang RZ, Lee MJ, Glynn NM, Yu DZ, Pray J, Ndubuizu K, Patil S, Schwartz A, Kligman M: Omentin plasma levels and gene expression are decreased in obesity. Diabetes, 2007; 56: 1655

9) Pan HY, Lin G, Li Q: Changes of serum omentin-1 levels in normal subjects and in patients with impaired glucose regulation and with newly diagnosed and untreated type 2 diabetes. Diabetes Research \& Clinical Practice, 2010; 88: 29

10) Shibata R, Ouchi N, Kikuchi R, Takahashi R, Takeshita K, Kataoka Y, Ohashi K, Ikeda N, Kihara S, Murohara T: Circulating omentin is associated with coronary artery disease in men. Atherosclerosis, 2011; 219: 811-814

11) Du Y, Ji Q, Cai L, Huang F, Lai Y, Liu Y, Yu J, Han B, Zhu E, Zhang J, Zhou Y, Wang Z, Zhao Y: Association between omentin-1 expression in human epicardial adipose tissue and coronary atherosclerosis. Cardiovasc Diabetol, 2016; 15
12) Kadoglou NPE, Tahmatzidis DK, Giannakoulas C, Kapelouzou A, Gkontopoulos A, Parissis J, Lampropoulos S, Kottas G: Serum levels of novel adipokines, omentin-1 and chemerin, in patients with acute myocardial infarction. J Cardiovasc Med, 2015; 16: 341-346

13) Watanabe K, Watanabe R, Konii H, Shirai R, Sato K, Matsuyama T, Ishibashi-Ueda H, Koba S, Kobayashi Y, Hirano T, Watanabe T: Counteractive effects of omentin-1 against atherogenesis. Cardiovasc Res, 2016; 110: 118-128

14) Hiramatsu-Ito M, Shibata R, Ohashi K, Uemura $Y$, Kanemura N, Kambara T, Enomoto T, Yuasa D, Matsuo K, Ito M, Hayakawa S, Ogawa H, Otaka N, Kihara S, Murohara T, Ouchi N: Omentin attenuates atherosclerotic lesion formation in apolipoprotein E-deficient mice. Cardiovasc Res, 2016; 110: 107-117

15) Xu T, Zuo P, Cao L, Gao Z, Ke K: Omentin-1 is Associated with Carotid Plaque Instability among Ischemic Stroke Patients. J Atheroscler Thromb, 2018; 25: 505-511

16) Dohi T, Maehara A, Brener SJ, Généreux P, Gershlick AH, Mehran R, Gibson CM, Mintz GS, Stone GW: Utility of Peak Creatine Kinase-MB Measurements in Predicting Myocardial Infarct Size, Left Ventricular Dysfunction, and Outcome After First Anterior Wall Acute Myocardial Infarction (from the INFUSE-AMI Trial). Am J Cardiol, 2015; 115: 563-570

17) Richards AM, Nicholls MG, Yandle TG, Frampton C, Espiner EA, Turner JG, Buttimore RC, Lainchbury JG, Elliott JM, Ikram H: Plasma N-Terminal Pro-Brain Natriuretic Peptide and Adrenomedullin. Circulation, 1998; 97: 1921-1929

18) Talwar S: Profile of plasma N-terminal proBNP following acute myocardial infarction. Correlation with left ventricular systolic dysfunction. Eur Heart J, 2000; 21: 15141521

19) Ohashi K, Shibata R, Murohara T, Ouchi N: Role of antiinflammatory adipokines in obesity-related diseases. Trends Endocrin Met, 2014; 25: 348-355

20) Tan YL, Zheng XL, Tang CK: The protective functions of omentin in cardiovascular diseases. Clin Chim Acta, 2015; 448: 98-106

21) Saddic LA, Nicoloro SM, Gupta OT, Czech MP, Gorham J, Shernan SK, Seidman CE, Seidman JG, Aranki SF, Body SC, Fitzgibbons TP, Muehlschlegel JD: Joint analysis of left ventricular expression and circulating plasma levels of Omentin after myocardial ischemia. Cardiovasc Diabetol, 2017; 16

22) Alkuraishy HM, Algareeb AI: New Insights into the Role of Metformin Effects on Serum Omentin-1 Levels in Acute Myocardial Infarction: Cross-Sectional Study. Emergency Medicine International, 2015; 2015: 283021

23) Moreno-Navarrete JM, Catalán V, Ortega F, GómezAmbrosi J, Ricart W, Frühbeck G, Fernández-Real JM: Circulating omentin concentration increases after weight loss. Nutr Metab, 2010; 7: 27

24) Chen Q, Shang X, Yuan M, Liang L, Zhong X: Effect of atorvastatin on serum omentin-1 in patients with coronary artery disease. Coronary Artery Dis, 2017; 28: 44-51

25) Derosa G, Cicero AFG, Franzetti IG, Querci F, Carbone A, Ciccarelli L, D Angelo A, Fogari E, Maffioli P: Effects of exenatide and metformin in combination on some adi- 
pocytokine levels: a comparison with metformin monotherapy. Can J Physiol Pharm, 2013; 91: 724-732

26) Yan P, Li L, Yang M, Liu D, Liu H, Boden G, Yang G: Effects of the long-acting human glucagon-like peptide-1 analog liraglutide on plasma omentin-1 levels in patients with type 2 diabetes mellitus. Diabetes Res Clin Pr, 2011; 92: 368-374

27) Frangogiannis NG, Lindsey ML, Michael LH, Youker KA, Bressler RB, Mendoza LH, Spengler RN, Smith CW, Entman ML: Resident cardiac mast cells degranulate and release preformed TNF-alpha, initiating the cytokine cascade in experimental canine myocardial ischemia/reperfusion. Circulation, 1998; 98: 699-710

28) Frangogiannis NG, Smith CW, Entman ML: The inflammatory response in myocardial infarction. Cardiovasc Res, 2002; 53: 31-47

29) Trifunovic D, Stankovic S, Marinkovic J, Beleslin B, Banovic M, Djukanovic N, Orlic D, Tesic M, Vujisic-Tesic B, Petrovic $M$, Nedeljkovic I, Stepanovic J, Djordjevic-Dikic A, Giga V, Ostojic M: Time-dependent changes of plasma adiponectin concentration in relation to coronary microcirculatory function in patients with acute myocardial infarction treated by primary percutaneous coronary intervention. J Cardiol, 2015; 65: 208-215

30) Natsukawa T, Maeda N, Fukuda S, Yamaoka M, Fujishima Y, Nagao H, Sato F, Nishizawa H, Sawano H, Hayashi Y: Significant Association of Serum Adiponectin and Creatine Kinase-MB Levels in ST-Segment Elevation Myocardial Infarction. J Atheroscler Thromb, 2017; 24: 793803

31) Matsuda K, Fujishima Y, Maeda N, Mori T, Hirata A, Sekimoto R, Tsushima Y, Masuda S, Yamaoka M, Inoue K, Nishizawa H, Kita S, Ranscht B, Funahashi T, Shimomura I, Adiponectin Adipo AMAP: Positive feedback regulation between adiponectin and T-cadherin impacts adiponectin levels in tissue and plasma of male mice. Endocrinology, 2015; 156: 934-946
32) Zhao D, Feng P, Sun Y, Qin Z, Zhang Z, Tan Y, Gao E, Lau WB, Ma X, Yang J, Yu S, Xu X, Yi D, Yi W: Cardiacderived CTRP9 protects against myocardial ischemia/reperfusion injury via calreticulin-dependent inhibition of apoptosis. Cell Death Dis, 2018; 9

33) Narumi T, Watanabe T, Kadowaki S, Kinoshita D, Yokoyama M, Honda Y, Otaki Y, Nishiyama S, Takahashi H, Arimoto T: Impact of serum omentin-1 levels on cardiac prognosis in patients with heart failure. Cardiovasc Diabetol, 2014; 13: 84

34) Yamawaki H, Tsubaki N, Mukohda M, Okada M, Hara Y: Omentin, a novel adipokine, induces vasodilation in rat isolated blood vessels. Biochem Bioph Res Co, 2010; 393: 668-672

35) Maruyama S, Shibata R, Kikuchi R, Izumiya Y, Rokutanda T, Araki S, Kataoka Y, Ohashi K, Daida H, Kihara S, Ogawa H, Murohara T, Ouchi N: Fat-derived Factor Omentin Stimulates Endothelial Cell Function and Ischemia-induced Revascularization via Endothelial Nitric Oxide Synthase-dependent Mechanism. J Biol Chem, 2011; 287: 408-417

36) Yamawaki H, Kuramoto J, Kameshima S, Usui T, Okada M, Hara Y: Omentin, a novel adipocytokine inhibits TNFinduced vascular inflammation in human endothelial cells. Biochem Bioph Res Co, 2011; 408: 339-343

37) Zhong X, Li X, Liu F, Tan H, Shang D: Omentin inhibits TNF- $\alpha$-induced expression of adhesion molecules in endothelial cells via ERK/NF- $\kappa$ B pathway. Biochem Bioph Res Co, 2012; 425: 401-406

38) Wang Y, Sun M, Wang Z, Li X, Zhu Y, Li Y: Omentin-1 ameliorates the attachment of the leukocyte THP-1 cells to HUVECs by targeting the transcriptional factor KLF2. Biochem Bioph Res Co, 2018; 498: 152-156

39) Rohrbach S, Troidl C, Hamm C, Schulz R: Ischemia and reperfusion related myocardial inflammation: A network of cells and mediators targeting the cardiomyocyte. Iubmb Life, 2015; 67: 110-119 